\title{
NOT EVERY $d$-SYMMETRIC OPERATOR IS GCR
}

\author{
C. RAY ROSENTRATER ${ }^{1}$
}

\begin{abstract}
Let $T$ be an element of $\mathscr{B}(\mathcal{F C})$, the algebra of bounded linear operators on the Hilbert space $\mathcal{H}$. The derivation induced by $T$ is the map $\delta_{T}(X)=T X-$ $X T$ from $\mathscr{B}(\mathscr{H})$ into itself. $T$ is $d$-symmetric if the norm closure of the range of $\delta_{T}$, $\Re\left(\delta_{T}\right)^{-}$, is closed under taking adjoints. This paper answers the question of whether every $d$-symmetric operator is GCR by giving an example of an NGCR weighted shift that is also $d$-symmetric.
\end{abstract}

Let $\mathcal{H}$ be a complex Hilbert space and $T$ an element of $\mathscr{B}(\mathcal{H})$, the algebra of bounded linear operators from $\mathcal{H}$ into $\mathcal{H}$. The derivation induced by $T$ is the mapping $\delta_{T}(X)=T X-X T$ from $\mathscr{B}(\mathcal{H})$ into itself. $T$ is said to be $d$-symmetric if the norm closure of the range of $\delta_{T}, \Re\left(\delta_{T}\right)^{-}$, is closed under taking adjoints. Examples of $d$-symmetric operators include the normal operators and isometries.

In [ABDW] it is proved that a necessary and sufficient condition for $T$ to be $d$-symmetric is that $T T^{*}-T^{*} T \in \mathcal{C}(T)$ where $\mathcal{C}(T)=\{C \in \mathscr{B}(\mathcal{H}): C \mathscr{B}(\mathcal{H})+$ $\left.\mathscr{B}(\mathcal{H}) C \subseteq \Re\left(\delta_{T}\right)^{-}\right\}$. In the same paper the question is raised whether every $d$-symmetric operator is GCR. This paper answers that question in the negative by giving an example of a weighted shift $T e_{i}=\alpha_{i} e_{i+1}, i \in \mathrm{Z}$, that is both $d$-symmetric and NGCR. Recall that an operator $T$ is GCR if every irreducible representation of $C^{*}(T)$, the $C^{*}$-algebra generated by $T$ and the identity operator, contains the compact operators. $T$ is NGCR if $C^{*}(T)$ contains no GCR two sided ideal [A]. If $T$ is irreducible then $T$ is NGCR if and only if $C^{*}(T)$ contains no nonzero compact operator [A].

LemMa. Let $V$ be similar to $T$, say $S V S^{-1}=T$. Then $T$ is $d$-symmetric if and only if $S^{-1}\left(T T^{*}-T^{*} T\right) S \in \mathcal{C}(V)$.

Proof. $\delta_{T}\left(S X S^{-1}\right)=S V X S^{-1}-S X V S^{-1}=S \delta_{V}(X) S^{-1}$. Hence $\Re\left(\delta_{T}\right)^{-}=$ $S \Re\left(\delta_{V}\right)^{-} S^{-1}$ and it follows that $\mathcal{C}(V)=S^{-1} \mathcal{C}(T) S$. Thus $C=T T^{*}-T^{*} T \in$ $\mathcal{C}(T)$ if and only if $S^{-1} C S \in \mathcal{C}(V)$. The lemma now follows from the result quoted above.

We now restrict our attention to weighted shifts. Recall that two bilateral shifts $V e_{i}=\alpha_{i} e_{i+1}$ and $T f_{i}=\beta_{i} f_{i+1}$ are similar if and only if there exist integer $k$ and constant $C$ so that $1 / C \leqslant\left|\left(\alpha_{k} \alpha_{k+1} \cdots \alpha_{k+n}\right) /\left(\beta_{0} \beta_{1} \cdots \beta_{n}\right)\right|<C$ uniformly for

Received by the editors March 13, 1980.

1980 Mathematics Subject Classification. Primary 47B47; Secondary 47A67, 47A05.

${ }^{1}$ The following material is to be contained in a dissertation written under the direction of $J$. P. Williams in partial fulfillment of the requirements of the $\mathrm{Ph}$. $\mathrm{D}$. degree at Indiana University. The author is grateful to J. P. Williams for his many helpful comments in the course of writing this manuscript. 
all $n \in Z$ (see [S]). If we define $T_{0} e_{i}=\beta_{i-k} e_{i+1}$ then $T_{0}$ is unitarily equivalent to $T, T_{0}$ is similar to $V$, and the similarity can be implemented by an operator that is diagonal with respect to $\left\{e_{n}\right\}$ (see [S]). The same results are true in the unilateral case with $k=0, n \in \mathbf{N}$. This leads to the following.

COROLlaRY. Let $V$ and $T$ be similar (unilateral or bilateral) weighted shifts. Then $T$ is d-symmetric if and only if $T_{0} T_{0}^{*}-T_{0}^{*} T_{0} \in \mathcal{C}(V)$.

Proof. Since $d$-symmetry is clearly preserved under unitary equivalence, $T$ is $d$-symmetric if and only if $T_{0}$ is $d$-symmetric. $T_{0}$ is similar to $V$ by means of a diagonal operator $D . T_{0} T_{0}^{*}-T_{0}^{*} T_{0}$ is diagonal with respect to the same basis so $D^{-1}\left(T_{0} T_{0}^{*}-T_{0}^{*} T_{0}\right) D=T_{0} T_{0}^{*}-T_{0}^{*} T_{0}$.

REMARK. If $S$ is an invertible operator that commutes with $C$, then it is not hard to show that $C \in \mathcal{C}(T)$ if and only if $C S \in \mathcal{C}(T)$. In particular, if $C=T_{0} T_{0}^{*}-$ $T_{0}^{*} T_{0}$ is a diagonal operator as in the corollary, then $C \in \mathcal{C}(T)$ if and only if $|C|$, the diagonal with diagonal entries the modulus of the corresponding entry in $C$, is in $\mathcal{C}(T)$. (It is not true in general that $|C| \in \mathcal{C}(T)$ implies $C \in \mathcal{C}(T)$.)

In [ABDW] it is shown that when $T$ is $d$-symmetric, $\mathcal{C}(T)$ is the linear span of the positive elements in $\Re\left(\delta_{T}\right)^{-}$. This implies the following.

Proposition. If $V$ is a d-symmetric weighted shift and $T$ is a weighted shift similar to $V$, then $T$ is d-symmetric if and only if $\left|T_{0} T_{0}^{*}-T_{0}^{*} T_{0}\right| \in R\left(\delta_{V}\right)^{-}$.

Before we proceed to the example, we need to state a result due to O'Donovan. In [O] he proves that a bilateral shift with nonzero weights $\{w(i)\}$ is NGCR if and only if there exists a sequence $n_{k} \rightarrow \infty$, such that $w\left(i+n_{k}\right) \rightarrow w(i)$ for $i \in \mathbf{Z}$.

EXAMPLE. Let $T$ be the bilateral weighted shift with weights defined by

$$
w(i)= \begin{cases}1, & i<0, \\ \frac{1}{2}, & i=1, \\ 2, & i=2, \\ 1, & 3^{k}<i<2 \cdot 3^{k} \\ w\left(i-2 \cdot 3^{k}\right), & 2 \cdot 3^{k}<i<3^{k+1}\end{cases}
$$

Claim I. $T$ is NGCR.

Proof. Let $n_{k}=2 \cdot 3^{k}$. Fix $i<0$. Then for $k>1$ so that $3^{k}>|i|, w\left(i+n_{k}\right)=$ $w\left(2 \cdot 3^{k}-|i|\right)=1=w(i)$.

Fix $i>0$. Then for $k$ so that $3^{k}>i$ we have $2 \cdot 3^{k}<i+2 \cdot 3^{k}<3^{k+1}$ so $w\left(i+n_{k}\right)=w\left(i+2 \cdot 3^{k}\right)=w(i)$. In any case we have $w\left(i+n_{k}\right) \rightarrow w(i)$.

Claim II. $T$ is similar to the bilateral shift $V e_{n}=e_{n+1}$ and $T_{0}=T$.

Proof. An induction argument shows that if $w(k)=2$ then $w(k-1)=\frac{1}{2}$ and if $w(k)=\frac{1}{2}$ then $w(k+1)=2$. Since all other weights are 1 it follows that

$$
\frac{1}{2}<|w(0) \cdot w(1) \cdot \cdots \cdot w(n)|<2 \text { for } n \in \mathbf{Z} \text {. }
$$


Matrix computations show that $D=\left|T T^{*}-T^{*} T\right|$ is the diagonal operator with the weights

$$
d(i)= \begin{cases}0, & i<0, \\ \frac{3}{4}, & i=1, \\ \frac{15}{4}, & i=2, \\ 3, & i=3, \\ 0, & 3^{k}<i<2 \cdot 3^{k} \\ d\left(i-2 \cdot 3^{k}\right), & 2 \cdot 3^{k}<i \leqslant 3^{k+1}\end{cases}
$$

In order to show $T$ is $d$-symmetric it is enough to show that $D=\left|T T^{*}-T^{*} T\right| \in$ $\Re\left(\delta_{V}\right)^{-}$by the proposition. As

$$
\delta_{V}\left(-\sum_{j=0}^{n-1}\left(\frac{n-j}{n}\right) V^{j} D V^{*(j+1)}\right)=D-\frac{1}{n} \sum_{j=1}^{n} V^{j} D V^{* j},
$$

we will be done if we show $3^{-k}\left\|\sum_{j=1}^{3^{k}} V^{j} D V^{* j}\right\| \rightarrow 0$ as $k \rightarrow \infty$.

Since conjugation by $V$ shifts a diagonal operator one position down the diagonal, $\sum_{j=1}^{n} V^{j} D V^{* j}$ is also a diagonal operator and its weights are $d^{\prime}(i)=$ $\sum_{j=1}^{n} d(i-j)=\sum_{j=1}^{n} d(i-n+j)$. Thus it suffices to show that

$$
\frac{1}{3^{k}} \sum_{j=1}^{3^{k}} d(i+j) \rightarrow 0 \text { uniformly in } i \text { as } k \rightarrow \infty \text {. }
$$

ClaIm III. $\sum_{j=1}^{3^{k}} d(j)<8 \cdot 2^{k}$.

Proof. If $k=1$, then $\Sigma_{j=1}^{3} d(j)=15 / 2<8$. Assuming $\Sigma_{j=1}^{3^{k}} d(j)<8 \cdot 2^{k}$ we see that

$$
\begin{aligned}
\sum_{j=1}^{3^{k+1}} d(j) & =\sum_{j=1}^{3^{k}} d(j)+\sum_{j=3^{k}+1}^{2 \cdot 3^{k}} d(j)+\sum_{j=2 \cdot 3^{k}+1}^{3^{k+1}} d(j) \\
& =2 \sum_{j=1}^{3^{k}} d(j)<8 \cdot 2^{k+1} \cdot \square
\end{aligned}
$$

Claim IV. $\Sigma_{j=1}^{3^{\prime}} d(i+j)<8 \cdot 2^{l}$ for all $i \in \mathbf{Z}$.

Proof. Suppose that $-\infty<i<3^{l}$. Since $d(j)=0$ for $j<0$ and $3^{l}<j \leqslant 2 \cdot 3^{l}$,

$$
\begin{aligned}
\sum_{j=1}^{3^{\prime}} d(i+j) & =\sum_{j=i+1}^{3^{l}+i} d(j)<\sum_{j=1}^{2 \cdot 3^{\prime}} d(j) \\
& =\sum_{j=1}^{3^{l}} d(j)<8 \cdot 2^{l}
\end{aligned}
$$

by Claim III.

Let $k \geqslant l$ and assume that $\sum_{j=1}^{3^{\prime}} d(i+j)<8 \cdot 2^{l}$ for $i<3^{k}$. Let $3^{k}<i<3^{k+1}$ and consider

$$
\sum_{j=1}^{3^{\prime}} d(i+j)=\sum_{j=i+1}^{i+3^{\prime}} d(j)
$$


If $i+3^{l} \leqslant 2 \cdot 3^{k}$ then the sum is zero since $d(j)=0$ for $3^{k}<j<2 \cdot 3^{k}$. For the same reason we can assume that the lower limit on the sum is at least $2 \cdot 3^{k}$. Since $d(j)=0$ for $3^{k+1}<j \leqslant 3^{k+1}+3^{l}<2 \cdot 3^{k+1}$, we can also assume that the upper limit is at most $3^{k+1}$. Hence $\sum_{j=i+1}^{i+3^{l}} d(j)=\sum_{j=n+1}^{m} d(j)$ with $2 \cdot 3^{k} \leqslant n<m<3^{k+1}$ and $m-n \leqslant 3^{l}$. Let $m^{\prime}=m-2 \cdot 3^{k}$ and $n^{\prime}=n-2 \cdot 3^{k}$; then we see that

$$
\sum_{j=n+1}^{m} d(j)=\sum_{j=n^{\prime}+1}^{m^{\prime}} d(j)<\sum_{j=n^{\prime}+1}^{n^{\prime}+3^{l}} d(j)<8 \cdot 2^{l}
$$

since $n^{\prime} \leqslant 3^{k+1}-2 \cdot 3^{k}=3^{k}$. Hence

$$
\sum_{j=1}^{3^{\prime}} d(i+j)<8 \cdot 2^{l} \text { for } i<3^{k+1} .
$$

Thus we have shown that $T$ is both NGCR and $d$-symmetric.

\section{REFERENCES}

[An] J. Anderson, On normal derivations, Proc. Amer. Math. Soc. 38 (1973), 135-140.

[ABDW] J. Anderson, J. Bunce, J. Deddens and J. P. Williams, $C^{*}$-algebras and derivation ranges, Acta Sci. Math. 40 (1978), 211-227.

[A] W. Arveson, An invitation to $C^{*}$-algebras, Springer-Verlag, New York, Heidelberg and Berlin, 1976.

[BD] J. Bunce and J. Deddens, $C^{*}$-algebras generated by weighted shifts, Indiana Univ. Math. J. 23 (1973), 257-271.

[JW] B. E. Johnson and J. P. Williams, The range of a normal derivation, Pacific J. Math. 58 (1975), 105-122.

[O] D. O'Donovan, Weighted shifts and covariance algebras, Trans. Amer. Math. Soc. 208 (1975), $1-25$.

[S] A. Shields, Weighted shift operators and analytic function theory, Topics in Operator Theory, Math. Surveys, no. 13, Amer. Math. Soc., Providence, R. I., 1974, pp. 49-128.

[St] J. G. Stampfli, Derivations on $\mathscr{B}(\mathscr{H})$ : The range, Illinois J. Math. 17 (1973), 518-524.

[W1] J. P. Williams, On the range of a derivation, Pacific J. Math. 38 (1971), 273-279.

[W2] _ On the range of a derivation. II, Proc. Roy. Irish Acad. Sect. A 74 (1974), 299-310.

Department of Mathematics, Indiana University, Bloomington, Indiana 47401

Current address: Department of Mathematics, Westmont College, Santa Barbara, California 93108 